\title{
NOTES ON ACCURACY OF FINITE-VOLUME DISCRETIZATION SCHEMES ON IRREGULAR GRIDS
}

\author{
BORIS DISKIN* AND JAMES L. THOMAS ${ }^{\dagger}$
}

\begin{abstract}
Truncation-error analysis is a reliable tool in predicting convergence rates of discretization errors on regular smooth grids. However, it is often misleading in application to finitevolume discretization schemes on irregular (e.g., unstructured) grids. Convergence of truncation errors severely degrades on general irregular grids; a design-order convergence can be achieved only on grids with a certain degree of geometric regularity. Such degradation of truncation-error convergence does not necessarily imply a lower-order convergence of discretization errors. In these notes, irregular-grid computations demonstrate that the design-order discretization-error convergence can be achieved even when truncation errors exhibit a lower-order convergence or, in some cases, do not converge at all.
\end{abstract}

1. Introduction. These notes are a response to the recently published article [18]. The article applies a truncation-error analysis to evaluate accuracy of finitevolume discretization (FVD) schemes on general unstructured grids. The analysis is accompanied by computations performed on regular and irregular grids. While we agree with the analysis and its conclusions in application to regular smooth grids, we consider the truncation-error analysis and some of the conclusions derived from it invalid in application to irregular-grid computations.

On regular grids, convergence of truncation errors (also referred as local errors in the literature) is an accurate indicator of convergence of discretization errors (also referred as global errors). However, the truncation-error convergence is often misleading for FVD schemes defined on irregular (e.g., unstructured) grids. As shown in [18] and before this in [17], the second-order convergence of truncation errors for some commonly used FVD schemes can be achieved only on grids with a certain degree of geometric regularity. Other studies, e.g., $[3,6,7,10,13,14,15,16,20,21]$, showed that truncation-error convergence degradation on irregular grids does not necessarily imply a degradation of discretization-error convergence. Examples shown in the following sections confirm that on irregular grids, the design-order discretization-error convergence can be achieved even when truncation errors exhibit a lower-order convergence or, in some cases, do not converge at all. Note that these results do not contradict the Lax theorem, which states that consistency (convergence of truncation errors) and stability are sufficient (not necessary) for convergence of discretization errors. In fact, for some formally inconsistent discretization schemes, it has been rigorously proved that the discretization errors converge $[3,6,7,13,16,21]$.

Article [18] applied a truncation error analysis to FVD schemes for the Poisson equation. A thin-layer approximation was analyzed. It was shown that the truncation error is $O(1)$ (i.e., does not converge) in grid refinement unless the grids are regular. The discretization error of the scheme was inferred to be non-convergent. By coincidence, the particular thin-layer FVD scheme considered in [18] is indeed zeroth order accurate on general unstructured grids. The inaccuracy is caused by poor approximation to the normal gradients with the thin-layer approximation, not by $O(1)$ convergence of truncation errors.

\footnotetext{
*National Institute of Aerospace (NIA), 100 Exploration Way., Hampton, VA 23681 (bdiskin@nianet.org) also Mechanical \& Aerospace Engineering Department, University of Virginia, Charlottesville, VA 22902.

${ }^{\dagger}$ Computational Aerosciences Branch, NASA Langley Research Center, Mail Stop 128, Hampton, VA 23681-2199 (James.L.Thomas@nasa.gov).
} 
In [18], a general conclusion was drawn that "a compact finite volume approximation of the Laplacian has to rely on symmetries in the grid to be first-order accurate." This conclusion is incorrect. For example, a common finite-volume scheme equivalent to a Galerkin finite-element approximation on triangles satisfies the definition of a compact scheme and is known to have second-order discretization errors (and zeroth order truncation errors) on irregular (non-symmetric) grids. We show additional computations on general mixed-element grids demonstrating the second-order convergence of discretization errors and zeroth order convergence of truncation errors.

Article [18] also considered a central FVD scheme for an advection equation on mixed-element and perturbed quadrilateral grids. Truncation-error analysis showed a zeroth-order convergence in the $L_{\infty}$ norm. Supporting computations showed a zeroth order convergence of discretization errors. It was concluded that FVD schemes for an advection equation are non-convergent on non-smooth irregular grids. The conclusion is wrong because there are counter examples of FVD schemes with truncation errors that do not converge on general irregular grids but with discrete solutions that converge with at least first order in any norm. The numerical scheme considered in [18] is not representative of current practice - the central scheme is known to exhibit erratic convergence of discrete solutions because of checkerboard instabilities. Tests exposing the erratic convergence of discretization errors of the central scheme are shown in Section 4 of these notes.

In the following Section 2, we provide definitions and discuss relations between truncation and discretization errors. Section 3 presents simple one-dimensional examples that expose shortcomings of the truncation-error analysis for irregular-grid FVD schemes. Section 4 presents numerical two-dimensional computations for the Poisson equation on mixed-element grids and for an advection equation on perturbed quadrilateral grids similar to those used in [18].

2. Formulation. For completeness of the presentation, we start from a brief formulation of finite-volume discretization (FVD) schemes and definitions of the corresponding truncation and discretization errors. The FVD schemes are derived from the integral form of a steady conservation law

$$
\oint_{\Gamma}(\mathbf{F} \cdot \hat{\mathbf{n}}) d \Gamma=\iint_{\Omega} f d \Omega,
$$

where $f$ is a forcing function, $\Omega$ is a control volume with boundary $\Gamma, \hat{\mathbf{n}}$ is the outward unit normal vector, and $\mathbf{F}$ is the flux vector that may include viscous and/or inviscid contributions. The general FVD approach requires partitioning the domain into a set of non-overlapping control volumes and implementing numerically equation (2.1) over each control volume. In this paper, we are considering node-centered FVD schemes, where solution values are stored at the mesh nodes.

For two-dimensional (2D) node-centered FVD schemes, a median-dual partition is constructed by connecting the mass centers of the primal-mesh cells with the midpoints of the surrounding edges (Figure 2.1). These non-overlapping control volumes cover the entire computational domain and compose a mesh that is dual to the primal mesh. The discretization is applied at a sequence of refined grids composed of triangular and quadrilateral cells satisfying the consistent refinement property $[8,20]$. The property requires the maximum distance across the cells to decrease consistently with increase of the total number of grid points, $N$. In particular, the maximum distance should tend to zero as $N^{-1 / 2}$ in $2 \mathrm{D}$ computations. 


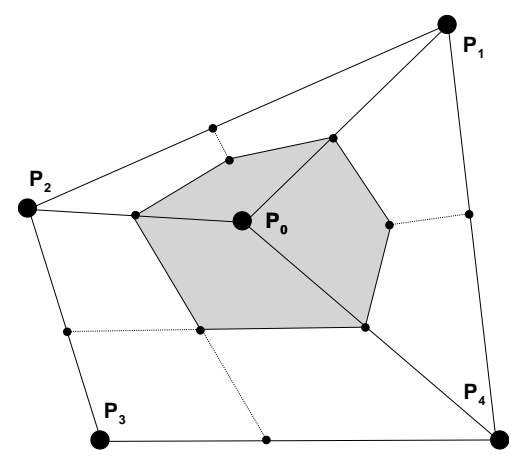

FIG. 2.1. Median-dual partition for node-centered finite-volume discretizations. $P_{0}-P_{4}$ denote grid nodes. The control volume (dual cell) around $P_{0}$ is shaded.

The main accuracy measure of any FVD scheme is the discretization error, $E_{d}$, defined as the difference between the exact discrete solution, $Q^{h}$, of the discretized equations (2.1) and the exact continuous solution, $Q$, to the differential conservation law

$$
\begin{gathered}
\nabla \mathbf{F}=f, \\
E_{d}=Q-Q^{h} ;
\end{gathered}
$$

$Q$ is sampled at grid nodes.

Truncation error, $E_{t}$, measures the accuracy of the discrete approximation to the differential equations (2.2). For FVD schemes, the traditional truncation error is usually defined from the time-dependent standpoint $[19,22]$. In the steady-state limit, it is defined (e.g., in [10]) as the residual computed after substituting $Q$ into the normalized discrete equations (2.1),

$$
E_{t}=\frac{1}{|\Omega|}\left[-\iint_{\Omega} f^{h} d \Omega+\oint_{\Gamma}\left(\mathbf{F}^{\mathbf{h}}(Q) \cdot \hat{\mathbf{n}}\right) d \Gamma\right],
$$

where $\mathbf{F}^{\mathbf{h}}$ is a reconstruction of the flux $\mathbf{F}$ at the boundary $\Gamma,|\Omega|$ is the measure of the control volume,

$$
|\Omega|=\iint_{\Omega} d \Omega,
$$

$f^{h}$ is an approximation of the forcing function $f$ on $\Omega$, and the integrals are computed according to some quadrature formulas.

For a given FVD scheme, we define a residual function

$$
R(q)=\frac{1}{|\Omega|}\left[\oint_{\Gamma}\left(\mathbf{F}^{\mathbf{h}}(q) \cdot \hat{\mathbf{n}}\right) d \Gamma-\iint_{\Omega} f^{h} d \Omega\right],
$$


where $q$ is a discrete function defined at the grid nodes. The residual accounts for interior discretization and all boundary conditions. Note that by definition

$$
R\left(Q^{h}\right)=0
$$

and

$$
R(Q)=E_{t} .
$$

Substituting (2.3) into (2.7),

$$
R\left(Q-E_{d}\right)=0,
$$

and assuming the discretization error to be small comparing to the exact continuous solution, $Q,\left(\left|E_{d}\right|<<|Q|\right)$, we obtain

$$
E_{t}-J(Q) E_{d} \approx 0,
$$

where $J(Q)$ is the Jacobian of $R(q)$ computed for $q=Q$,

$$
J(Q)=\frac{1}{|\Omega|} \frac{\partial}{\partial q}\left[\oint_{\Gamma}\left(\mathbf{F}^{\mathbf{h}}(Q) \cdot \hat{\mathbf{n}}\right) d \Gamma\right] .
$$

Thus, the discretization error can be estimated as

$$
E_{d} \approx J^{-1} E_{t},
$$

where the inverse Jacobian is a convolution operator with a kernel that usually possesses certain smoothness properties. The discretization error in a point can be approximated as a weighted sum of the undivided truncation errors,

$$
E_{d} \approx \sum_{j} w_{j}\left(E_{t}\right)_{j}\left|\Omega_{j}\right|,
$$

where summation index $j$ goes over all control volumes and weighting factors, $w_{j}$, are determined by the convolution kernel.

Two norms are used in this paper to evaluate magnitudes of the discrete errors: the $L_{\infty}$ norm (also referred as max-norm) is computed as the maximum absolute value of a discrete function; the $L_{1}$ norm is computed as the mean absolute value, i.e., the sum of the absolute values divided by the number of discrete entries. Thus, either norm of a constant discrete function is the same constant.

Irregular grid computations, in which convergence of discretization errors surpasses the convergence of truncation errors, were reported and analyzed in literature since the 1960 s (see, e.g., [3, 6, 7, 10, 13, 14, 15, 20, 21]). In [13], discretization schemes demonstrating such convergence were called supra-convergent. While a rigorous proof of discretization error convergence for FVD schemes on general irregular grids is not yet available, there are several recent publications addressing supra-convergence on irregular grids. Papers $[7,16]$ consider formally inconsistent (no truncation-error convergence) schemes for advection equations in $1 \mathrm{D}$ and $2 \mathrm{D}$ and prove convergence of discretization errors; Barbeiro [3] proves second-order convergence of discretization errors for "inconsistent" discretizations of $2 \mathrm{D}$ elliptic equations on nonuniform grids. 
A qualitative explanation of supra-convergence observed for FVD schemes on irregular grids was suggested by Giles [10]. Giles' arguments can be illustrated as follows. First consider a node-centered FVD scheme on a sequence of regular grids. On such grids, truncation and discretization errors would converge with the same design order. Now consider effects of a local irregularity introduced, for example, by shifting one node. The node shift leads to changes in faces (and corresponding fluxes) of the control volume containing this node and its neighboring control volumes. Fluxes outside of this immediate neighborhood remain unchanged. Each (changed) flux at an internal face contributes equal and opposite amounts to the undivided truncation errors on both sides of the face. Thus, the change introduced into the local truncation error can be large (the change in a flux divided by the control volume), but the mean value (and, often, higher moments) of the changes introduced into the undivided truncation errors is zero. If the convolution kernel is sufficiently smooth, e.g., for all nodes outside of the immediate neighborhood of the perturbed node, the weighting factors do not vary too much over the control volumes with perturbed truncation errors, then the discretization error changes are close to zero outside of this immediate neighborhood. Even within this neighborhood, the discretization error changes are small comparing with the changes in the truncation errors because of the averaging effect.

3. One-dimensional examples. Examples in this section show that common FVD schemes on one-dimensional (1D) irregular grids exhibit supra-convergence. Similar computations have been demonstrated in $[15,16]$.

3.1. Spatial discretization grids. A $1 D$ discretization grid is defined as a combination of the primal and dual nodes. The solution values are located at the primal nodes; the fluxes are located at the dual nodes. For node-centered discretizations, a natural strategy is to place the primal mesh first and, then, use this mesh as a reference for placing dual control volumes.

The discretization grids used in this section are designed to demonstrate effects of grid irregularities and are described as follows. The first and the last of the $N+1$ primal nodes, $x_{i}, i=0,1, \ldots, N$, are always located at the ends of the computational interval; the interior nodes can be distributed either uniformly or randomly. Either distribution retains the nodal ordering and ensures that the maximal distance between the neighboring nodes is $O(1 / N)$. Let $s_{i}, i=0,1, \ldots,(N+1)$ denote the flux locations. The first and the last fluxes are also located at the ends of the interval. The location of an interior flux, $s_{i}$, is always between the primal nodes $x_{i-1}$ and $x_{i}$ and initially defined as the midpoint; the dual node $s_{i}$ may then be randomly perturbed about the midpoint.

Specifically, on an interval $x \in[a, b]$, the primal nodes are distributed according to

$$
x_{0}=a ; \quad x_{i}=a+\left(i+r_{i}\right) \frac{b-a}{N}, \quad i=1, \ldots,(N-1) ; \quad x_{N}=b ;
$$

where $r_{i}$ is either zero (uniform primal mesh) or a random number $-0.4 \leq r_{i} \leq 0.4$ (random primal mesh). The dual-mesh nodes are computed accordingly as

$$
s_{0}=a ; \quad s_{i}=x_{i-1}+d_{i}\left(x_{i}-x_{i-1}\right), \quad i=1, \ldots, N ; \quad s_{N+1}=b ;
$$

where $d_{i}=0.5$ and $d_{i}=0.5+r_{i}^{s}$ correspond to unperturbed and perturbed dual meshes, respectively; here $r_{i}^{s}$ is a random number $-0.25 \leq r_{i}^{s} \leq 0.25$. Grid examples 


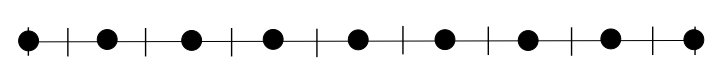

(a) Uniform primal mesh; unperturbed dual mesh.

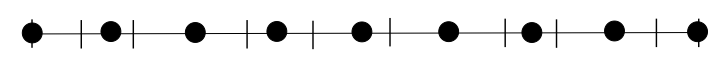

(b) Uniform primal mesh; perturbed dual mesh.

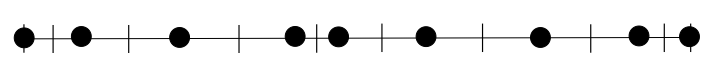

(c) Random primal mesh; unperturbed dual mesh.

FIG. 3.1. Examples of one-dimensional discretization grids: black bullets denote primal mesh nodes, vertical tic-marks denote dual mesh nodes.

are shown in Figure 3.1. The computations in this section refer to tests performed on the interval $x \in[0,1]$ using a sequence of grids with the total number of primal grid nodes increasing as $N=2^{n}+1 ; n=3,4, \ldots, 14$. All random grids are generated independently, so there is no regularity in the limit of grid refinement. The equivalent mesh size is taken as $h=\frac{1}{N}$.

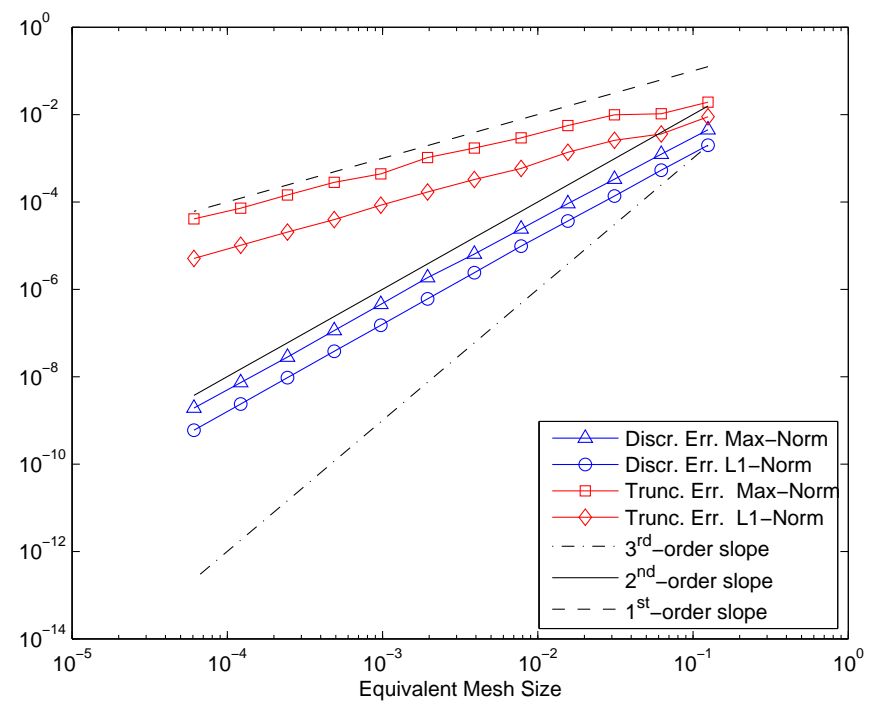

FIG. 3.2. Convergence of discretization and truncation errors in grid-refinement computations for the constant-coefficient convection equation. The tests are performed with random primal meshes and unperturbed dual meshes. 
3.2. Steady convection equation. The first example is a steady constantcoefficient convection equation

$$
\partial_{x} U=f(x), \quad U(0)=\psi,
$$

which is satisfied with the exact solution $U=\sin (x), \quad f=\cos (x)$. FVD equations are formed as follows

$$
F_{s_{i+1}}-F_{s_{i}}=\sin \left(s_{i+1}\right)-\sin \left(s_{i}\right), \quad i=0, \ldots, N .
$$

The fluxes, $F_{s_{i}}$, approximate solution values at the flux locations, $s_{i}$, and are computed by fully-upwind extrapolations from the primal nodes (except for the first interior dual node) as

$$
\begin{aligned}
& F_{s_{0}}=\psi, \\
& F_{s_{1}}=\frac{\left(s_{1}-x_{0}\right) U_{1}+\left(x_{1}-s_{1}\right) U_{0}}{x_{1}-x_{0}}, \\
& F_{s_{i}}=\frac{\left(s_{i}-x_{i-2}\right) U_{i-1}-\left(s_{i}-x_{i-1}\right) U_{i-2}}{x_{i-1}-x_{i-2}}, \quad i=2, \ldots, N+1
\end{aligned}
$$

where $U_{i}$ is a discrete approximation to $U\left(x_{i}\right)$. Boundary conditions are implemented weakly by defining the inflow flux. The forcing term is integrated exactly over the dual volume.

Figure 3.2 shows convergence rates obtained in grid-refinement on irregular discretization grids involving random primal meshes and unperturbed dual meshes. In either the $L_{\infty}$ or $L_{1}$ norm, truncation and discretization errors converge with different orders.

\begin{tabular}{|c|c|c|c|}
\hline Primal & Dual & \multicolumn{2}{|c|}{ Grid-refinement computations } \\
\cline { 3 - 4 } Mesh & Mesh & Discr. Error & Trunc. Error \\
\hline Uniform & Unperturbed & $O\left(h^{2}\right)$ & $O\left(h^{2}\right)$ \\
\hline Uniform & Perturbed & $O\left(h^{2}\right)$ & $O(h)$ \\
\hline Random & Unperturbed & $O\left(h^{2}\right)$ & $O(h)$ \\
\hline Random & Perturbed & $O\left(h^{2}\right)$ & $O(h)$ \\
\hline \multicolumn{2}{|c}{ TABLE 3.1} \\
\hline
\end{tabular}
tion.

Convergence of discretization and truncation errors for the constant-coefficient convection equa-

Table 3.1 summarizes discretization and truncation error convergence rates observed in computations. The results demonstrate that grid irregularity strongly affects the truncation-error convergence, but has no effect on the convergence order of the discretization errors.

3.3. Time dependent convection equation. The time-dependent constantcoefficient convection equation is defined on the domain $(t, x) \in[0,1] \times[0,1]$ as

$$
\partial_{t} U+\partial_{x} U=f(x), \quad U(t, 0)=\psi(t), \quad U(0, x)=\phi(x),
$$

and is satisfied with the exact solution $U(t, x)=\sin (2 \pi(x-t)), \quad f \equiv 0$. In computations, the number of time levels is equal to the number of spatial grid nodes, $N+1$. The time step is constant, $h_{t}=1 / N$. The same spatial grids are used at all time levels.

FVD equations at time level $k$ are formed as follows

$$
\left(-f_{i}^{k}+\partial_{t}^{h} U_{i}^{k}\right)\left(s_{i+1}-s_{i}\right)+F_{s_{i+1}}^{k}-F_{s_{i}}^{k}=0, \quad i=0, \ldots, N .
$$


Here, $\partial_{t}^{h} U_{i}^{k}$ is a discrete time derivative and $f_{i}^{k}$ is a discrete approximation to the forcing term; both are defined at grid node $x_{i}$. For $k>1, \partial_{t}^{h} U_{i}^{k}$ is the second-order backward difference scheme,

$$
\partial_{t}^{h} U_{i}^{k} \equiv \frac{1}{h_{t}}\left(\frac{3}{2} U_{i}^{k}-2 U_{i}^{k-1}+\frac{1}{2} U_{i}^{k-2}\right)
$$

and, for $k=1$, the time derivative is approximated by the first-order backward difference scheme,

$$
\partial_{t}^{h} U_{i}^{1} \equiv \frac{1}{h_{t}}\left(U_{i}^{1}-U_{i}^{0}\right)
$$

The spatial fluxes, $F_{s_{i}}^{k}$, are formed according to (3.5). The time dependent truncation error is evaluated for the exact solution, $U$, at each dual volume as

$$
E_{t}=-f_{i}^{k}+\partial_{t}^{h} U+\frac{F_{s_{i+1}}^{k}-F_{s_{i}}^{k}}{s_{i+1}-s_{i}}
$$

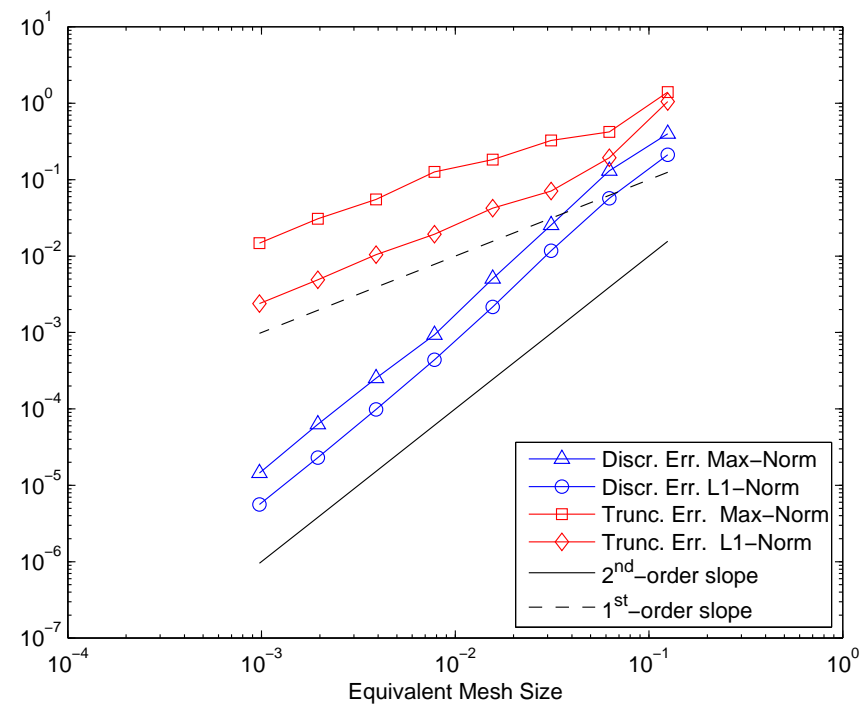

FIG. 3.3. Convergence of discretization and truncation errors in grid-refinement computations for the time-dependent constant-coefficient convection equation. The errors are computed at the final time $t=1$. The tests are performed with random primal meshes and unperturbed dual meshes.

Figure 3.3 shows convergence rates obtained in successive grid-refinement, which doubles the number of primal mesh intervals and time steps. Spatial grids involve random primal meshes and unperturbed dual meshes. The coarsest mesh includes 9 primal nodes ( 8 intervals) and uses 9 time levels ( 8 steps). Truncation and discretization errors are computed at the final time $t=1$. In either the $L_{\infty}$ or $L_{1}$ norm, the truncation errors converge with the first order and discretization errors converge with the second order. 
3.4. Diffusion equation. The third set of one-dimensional tests is performed for the constant-coefficient diffusion equation

$$
\partial_{x x} U=f(x), \quad U(0)=\psi_{0}, \quad U(1)=\psi_{1},
$$

which is defined on the interval $x \in[0,1]$, with the exact solution $U=\sin (x)$, $f=-\sin (x)$. FVD equations are formed at the interior dual volumes as

$$
F_{s_{i+1}}-F_{s_{i}}=\cos \left(s_{i+1}\right)-\cos \left(s_{i}\right), \quad i=1, \ldots, N .
$$

Fluxes approximating the solution derivative are defined as

$$
F_{s_{i}}=\frac{U_{i}-U_{i-1}}{x_{i}-x_{i-1}}, \quad i=1, \ldots, N
$$

where $U_{i}$ is a discrete approximation to $U\left(x_{i}\right)$ and the forcing term is integrated exactly. Dirichlet boundary conditions are strongly enforced as

$$
U_{0}=\psi_{0}, \quad U_{N+1}=\psi_{1} .
$$

\begin{tabular}{|c|c|c|c|}
\hline Primal & Dual & \multicolumn{2}{|c|}{ Grid-refinement computations } \\
\cline { 3 - 4 } Mesh & Mesh & Discr. Error & Trunc. Error \\
\hline Uniform & Unperturbed & $O\left(h^{2}\right)$ & $O\left(h^{2}\right)$ \\
\hline Uniform & Perturbed & $O\left(h^{2}\right)$ & $O(1)$ \\
\hline Random & Unperturbed & $O\left(h^{2}\right)$ & $O(h)$ \\
\hline Random & Perturbed & $O\left(h^{2}\right)$ & $O(1)$ \\
\hline \multicolumn{3}{|c}{ TABLE 3.2}
\end{tabular}

Convergence of discretization and truncation errors for the diffusion equation.

Table 3.2 summarizes the discretization and truncation error convergence. The main observation is that the discretization errors converge with second order even for those irregular grids for which truncation errors are $O(1)$.

4. Two-dimensional computations. In this section, we demonstrate $2 \mathrm{D}$ computations for the Poisson equation and for a constant-coefficient advection equation on general irregular grids. More details and other computations, including inviscid flow equations on general irregular grids, can be found in $[1,8,20]$.

4.1. Poisson equation. A widely used second-order FVD scheme is shown here for the Poisson equation,

$$
\Delta U=f,
$$

with Dirichlet boundary conditions. In the FVD scheme, the conservation law

$$
\oint_{\Gamma} \nabla \mathbf{U} \cdot \hat{\mathbf{n}} d \Gamma=\iint_{\Omega} f d \Omega
$$

is enforced on node-centered control volumes constructed by the median-dual partition; $\hat{\mathbf{n}}$ is the outward unit normal to the control-volume surface. In distinction from the thin-layer approximation considered in [18], the scheme uses the Green-Gauss 


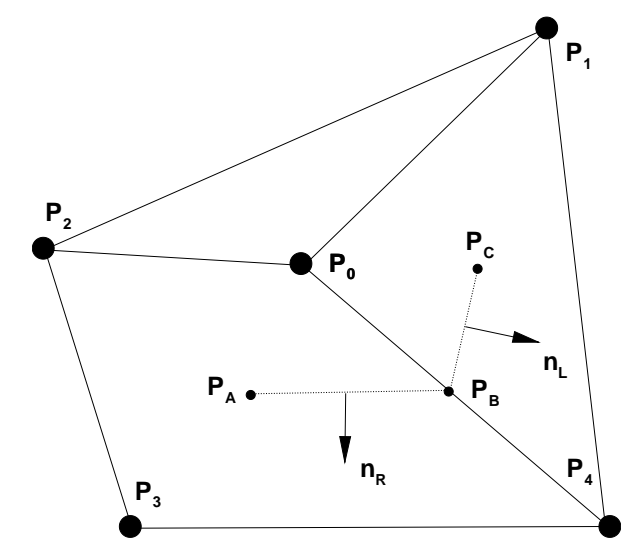

FIG. 4.1. Illustration of gradient reconstruction for viscous terms on mixed grids with mediandual partition.

approach to provide accurate approximations to the normal gradients at the controlvolume boundaries. A series of refined mixed-element grids composed of triangles and quadrangles is considered.

With reference to Figure 4.1, the integral flux through the dual faces adjacent to the edge $\left[P_{0}, P_{4}\right]$ is computed as

$$
\int_{A B C} \nabla \mathbf{U} \cdot \hat{\mathbf{n}} d \Gamma=\nabla \mathbf{U}^{R} \cdot \mathbf{n}_{\mathbf{R}}+\nabla \mathbf{U}^{L} \cdot \mathbf{n}_{\mathbf{L}},
$$

where $\mathbf{n}_{\mathbf{R}}$ and $\mathbf{n}_{\mathbf{L}}$ are directed areas of the corresponding dual faces. The gradient is reconstructed separately at each dual face as follows.

For the triangular element contribution, the gradient is determined from a GreenGauss evaluation at the primal-grid element,

$$
\nabla \mathbf{U}^{L}=\overline{\nabla \mathbf{U}}_{014}
$$

The gradient overbar denotes a gradient evaluated by the Green-Gauss formula on the primal cell identified by the point subscripts. With fully-triangular elements, the formulation is equivalent to a Galerkin finite element scheme with a linear basis function $[2,4]$. For the quadrilateral element contribution, the gradient is evaluated as

$$
\nabla \mathbf{U}^{R}=\bar{\nabla}_{0234}+\left[\frac{U_{4}-U_{0}}{\left|\mathbf{r}_{4}-\mathbf{r}_{0}\right|}-\bar{\nabla}_{0234} \cdot \mathbf{e}_{04}\right] \mathbf{e}_{04},
$$

where $\mathbf{r}_{i}$ is the coordinate vector of node $P_{i}$ and

$$
\mathbf{e}_{04}=\frac{\mathbf{r}_{4}-\mathbf{r}_{0}}{\left|\mathbf{r}_{4}-\mathbf{r}_{0}\right|}
$$

is the unit vector aligned with the edge $\left[P_{0}, P_{4}\right]$. Note that for grids with dual faces perpendicular to the edges, the edge-gradient is the only contributor. This approach to the gradient reconstruction is used to decrease the scheme susceptibility to oddeven decoupling $[11,12]$. In all cases, the linear solution reconstruction leads to a first-order flux (gradient) reconstruction accuracy. 


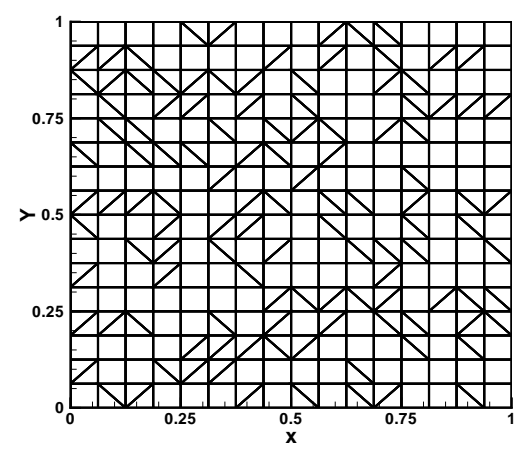

(a) A representative $17 \times 17$ mixed-element unstructured grid.

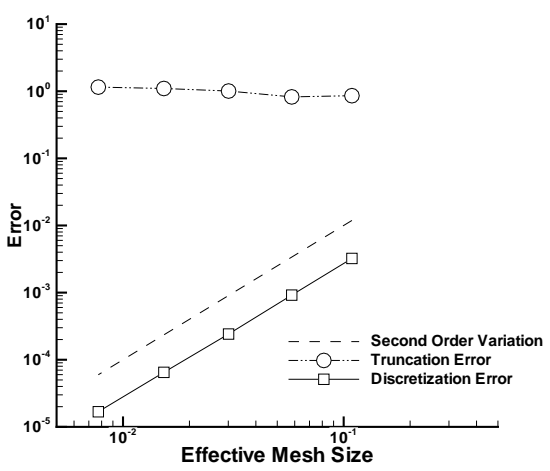

(b) Convergence of the discretization and truncation errors.

FIG. 4.2. Convergence of the discretization and truncation errors for the Poisson equation solved on mixed-element unstructured grids.

A sequence of globally refined mixed-element grids is generated on the unit square $(x, y) \in[0,1] \times[0,1]$ with $9,17,33,65$, and 129 points in the horizontal and vertical directions. Each grid is formed from an underlying Cartesian grid. Grid irregularity is enforced independently on each grid by random splitting (or not splitting) Cartesian cells into triangles; approximately half of the quadrangles are split. Figure 4.2(a) shows a representative $17 \times 17$ grid.

The exact solution is taken as $U=\sin (\pi x)+\sin (\pi y)$, and the corresponding forcing term is $f=-\pi^{2}[\sin (\pi x)+\sin (\pi y)]$. The $L_{1}$ norms of truncation and discretization errors are shown in Figure 4.2(b) versus an effective mesh size, taken as the $L_{1}$ norm of the square root of the dual volume. Discretization errors converge with the design second order; truncation errors do not converge. Although not shown, the error convergence rates in the $L_{\infty}$ norm are the same as the $L_{1}$-norm rates. On regular (either triangular or quadrilateral) meshes, both the truncation errors and the discretization errors converge with second order. In [18], $O(1)$ convergence of truncation errors on irregular grids was interpreted as an indication that the corresponding discrete solutions do not approximate the continuous ones; this example clearly shows that such inference can be wrong. The $O(1)$ convergence of the thin-layer scheme discretization errors shown in [18] is caused by poor approximation to the normal gradients provided by edge-derivatives on skewed grids, not by $O(1)$ convergence of truncation errors.

4.2. Advection equation. A constant coefficient $2 \mathrm{D}$ advection equation

$$
(\mathbf{a} \cdot \nabla) U=f
$$

is defined on the unit square $(x, y) \in[0,1] \times[0,1] ; \mathbf{a}=(a, b)^{T}=(1,1)^{T}$ is a constant vector. The characteristic direction is defined as $\xi=(a x+b y) / c$ where $c^{2}=a^{2}+b^{2}$. The cross-characteristic direction is defined as $\eta=(-b x+a y) / c$.

There are three exact solutions considered:

$$
U_{1}=\sin (4 \pi c \xi) ; \quad f_{1}=4 \pi c^{2} \cos (4 \pi c \xi)
$$




$$
\begin{gathered}
U_{2}=c\left(\xi+\xi^{2} / 2\right) ; \quad f_{2}=c^{2}(1+\xi) . \\
U_{3}=\sin (\pi c \eta) ; \quad f_{3}=0 .
\end{gathered}
$$

The first two solutions have variation in the characteristic direction; the third solution has variation in the cross-characteristic direction only.

In the edge-reconstruction FVD schemes considered in this paper, the conservation law

$$
\oint_{\Gamma}(\mathbf{a} \cdot \hat{\mathbf{n}}) U d \Gamma=\iint_{\Omega} f d \Omega
$$

is again enforced on node-centered control volumes constructed by the median-dual partition. The forcing term integration over the control volume is approximated as the node value multiplied by the volume $|\Omega|$. The term edge-reconstruction emphasizes that the quadrature scheme used for computing the integrals over the dual-cell boundaries employs fluxes reconstructed at the midpoints of the edges connecting the grid nodes. The numerical flux through the faces adjacent to the edge $\left[P_{0}, P_{1}\right]$ is computed as

$$
\mathbf{F}^{\mathbf{h}}=(\mathbf{a} \cdot \mathbf{n}) \bar{U}
$$

where $\bar{U}$ is a solution reconstruction at the edge midpoint and $\mathbf{n}$ is the combineddirected-area vector $\mathbf{n}=\mathbf{n}_{\mathbf{L}}+\mathbf{n}_{\mathbf{R}}$, where $\mathbf{n}_{\mathbf{L}}$ and $\mathbf{n}_{\mathbf{R}}$ are outward normal (directedarea) vectors of the left and right segments, respectively; see Figure 4.3 for illustration. FVD schemes with first- and second-order upwind reconstructions, which are scalar counterparts to widely used Euler schemes $[1,2,4,5,10,11,12,14,17,20]$, are considered as well as the central scheme used in [18].

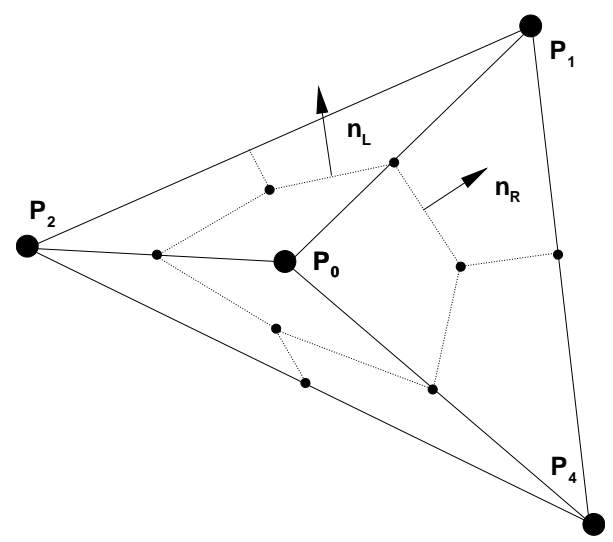

FIG. 4.3. Illustration for edge-reconstruction flux integration scheme in the interior.

The first-order upwind edge-reconstruction scheme approximates $\bar{U}$ from the solution at the upwind node. The second-order accurate upwind-biased (Fromm-type) edge-reconstruction scheme uses the least-square gradient at a node to reconstruct 
the solution at the edge midpoints. Second-order convergence of discretization errors has been demonstrated on general (e.g., irregular non-smooth) triangular grids. On irregular quadrilateral and mixed-element grids, the scheme accuracy deteriorates to first order; examples and explanations can be found in $[8,20]$.

Boundary conditions are enforced weakly through the boundary fluxes. At the inflow boundary, $\bar{U}$ is specified from the exact solution. At the outflow boundary, the boundary flux is determined from the solution value at the nearest node point, referred to here as a weak closure condition. This closure is approximately a first-order upwind differencing closure and is also that advocated in [18]. For triangular cells at the boundary, a special flux integrations scheme is applied to provide second-order accuracy; details can be found in $[2,8,20]$.

4.2.1. Perturbed quadrilateral grids. This section reports on investigation of the claim made in [18] that edge-reconstruction FVD schemes for perturbed quadrilaterals are zeroth order.

The considered uniform quadrilateral grids are Cartesian grids (Figure 4.4 (a)) defined on the domain $x \in[0,1]$ and $y \in[0,1]$. For the perturbed quadrilateral grids, all grid points are perturbed in both $x$ and $y$ directions by random shifts in the range $[-0.1,+0.1]$ of the local mesh size (Figure $4.4(\mathrm{~b})$ ). The sequences of globallyrefined grids are typically generated with $2^{n}+1$ points in both directions, where $n=3,4,5,6,7,8$.

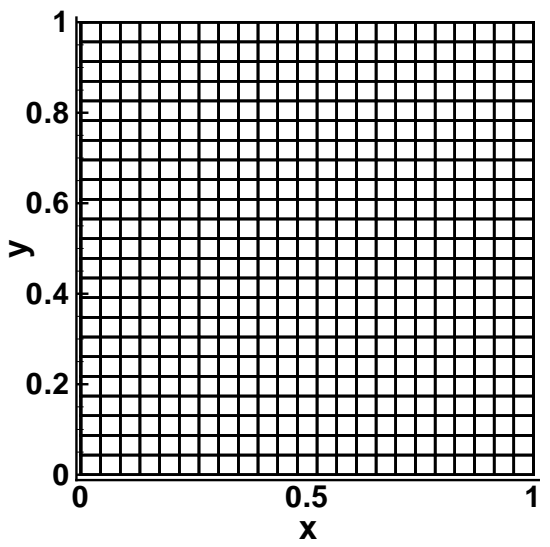

(a) Uniform grid.

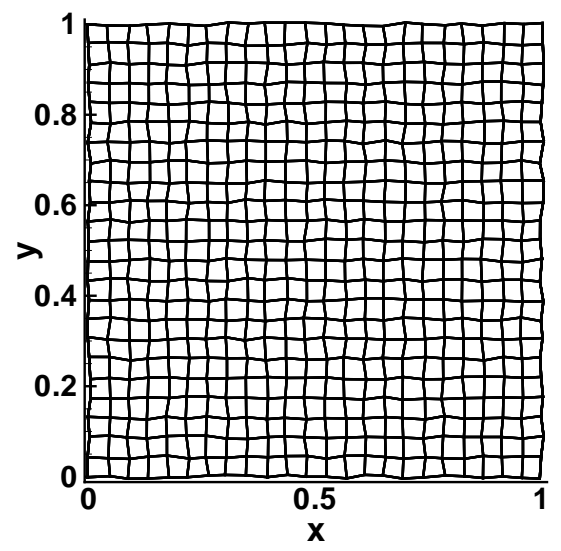

(b) Perturbed grid.

FIG. 4.4. Typical computational grid (shown with 24 points in each direction of the underlying Cartesian mesh).

In the figures that follow, the $L_{1}$ norms of truncation and discretization errors are shown versus an effective mesh size parameter, taken as the $L_{1}$ norm of the square root of the dual volume. The filled symbols denote truncation errors and the open symbols denote discretization errors. Circles, squares, and triangles refer to simulations with the first, second, and third exact solutions, respectively.

The results for the first-order accurate upwind edge-reconstruction scheme are shown in Figure 4.5. For the uniform grids, both truncation errors and discretization 


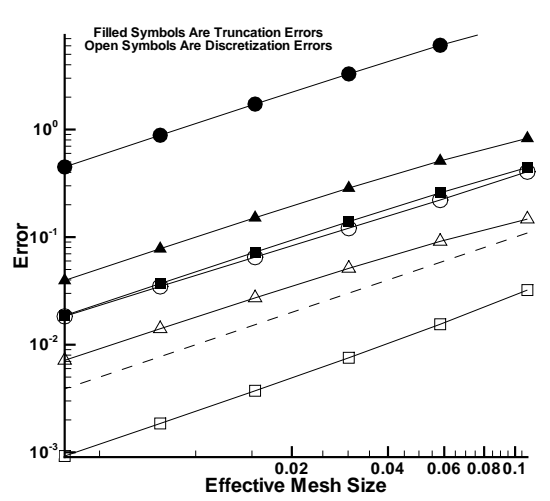

(a) Uniform grid.

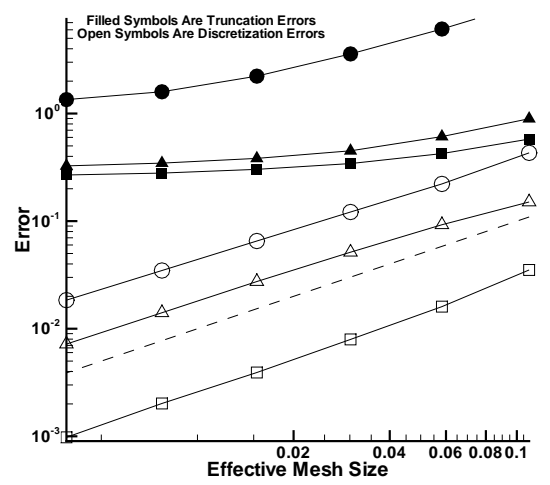

(b) Perturbed grid.

FIG. 4.5. Errors in grid refinement for first-order upwind edge-reconstruction scheme. Circles correspond to solution $U_{1}$, squares correspond to solution $U_{2}$, and triangles correspond to solution $U_{3}$. Dashed line indicates the first-order slope.

errors show a first-order convergence. For the perturbed grids, the truncation errors show one-order reduction in convergence, but the discretization errors remain firstorder accurate. Variations of the magnitude of the discretization error norm between the uniform and perturbed grids with the same number of points are very small.

Figure 4.6 shows the results for the second-order accurate upwind-biased (Frommtype) edge-reconstruction scheme. Again, for the uniform grids, both truncation errors and discretization errors show a second-order convergence. For the perturbed grids, the truncation errors show no convergence, i.e., zeroth-order convergence. On coarse grids, the discretization error convergence is better than first order. For solutions $U_{2}$ and $U_{3}$, the discretization errors asymptote clearly to first-order accuracy on finer grids. The results shown in Figures 4.5 and 4.6 clearly contradict to the conclusion drawn in [18] that FVD schemes that exhibit the zeroth order convergence of truncation errors are not convergent on irregular grids.

For illustration of erratic behavior of central schemes, the results for the secondorder accurate central scheme used in [18] are shown in Figure 4.7; this discretization reconstructs the solution by averaging the two nodal values on either side of an edge. This scheme is susceptible to checkerboard instabilities and boundary conditions are prominent in eliminating unstable modes. For the uniform grids, both truncation errors and discretization errors show a second-order convergence. Although not shown, the discretization errors exhibit a checkerboard pattern over the mesh.

For the perturbed grids, the truncation errors show no convergence, although we do not show their variation. The variation of the discretization errors is shown for two grid sequences. The sequences are derived from $9 \times 9$ and $13 \times 13$ coarsest grids. The discretization errors are quite erratic with grid refinement, as is typical of schemes with checkerboard instabilities. It might be inferred from the few coarsest grids that the discretization errors associated with $U_{2}$ and $U_{3}$ do not converge, but continued refinement shows that the discretization errors are converging, but apparently at a rate slower than first-order. 


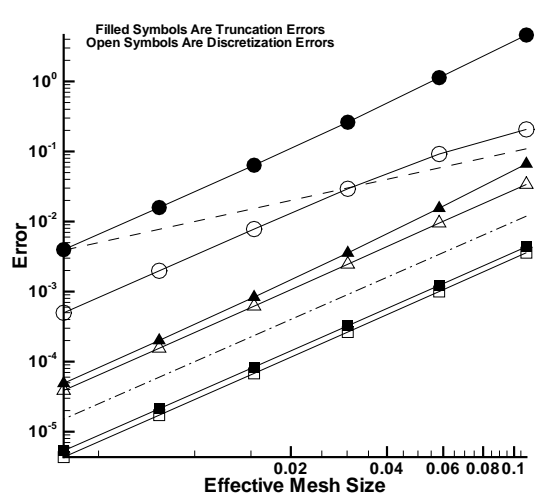

(a) Uniform grid.

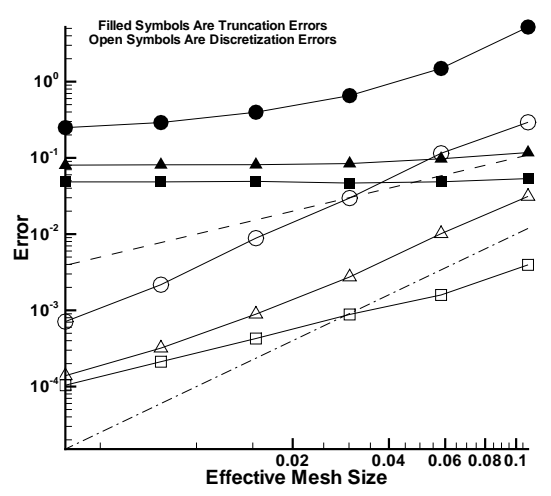

(b) Perturbed grid.

Fig. 4.6. Errors in grid refinement for second-order Fromm-type edge-reconstruction scheme. Circles correspond to solution $U_{1}$, squares correspond to solution $U_{2}$, and triangles correspond to solution $U_{3}$. Dashed and dotted lines indicate the first and second order slopes, respectively.

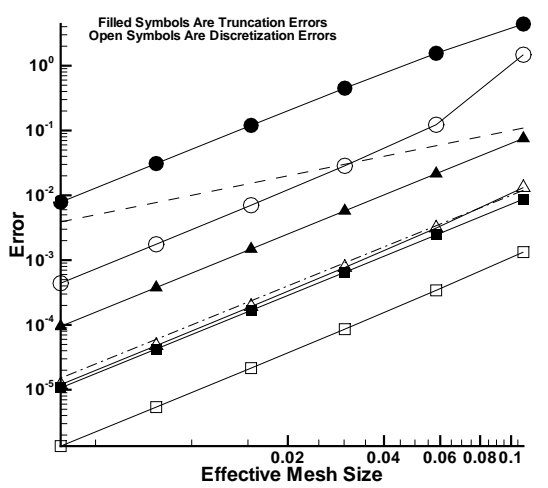

(a) Uniform grid.

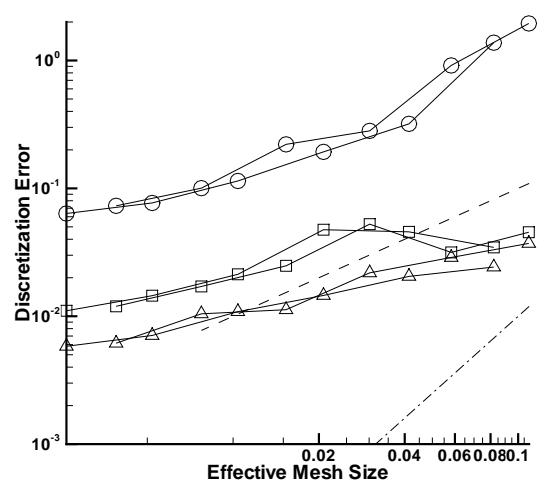

(b) Perturbed grid.

FIG. 4.7. Errors in grid refinement for second-order central edge-reconstruction scheme. Circles correspond to solution $U_{1}$, squares correspond to solution $U_{2}$, and triangles correspond to solution $U_{3}$. Dashed and dotted lines indicate the first and second order slopes, respectively.

Numerical results presented in this section show both the fully-upwind first-order edge-reconstruction scheme and an upwind-biased Fromm-type edge-reconstruction scheme to have first-order convergence of discretization errors on perturbed quadrilateral grids. These results are consistent with the results reported in $[1,8,20]$ for inviscid flow equations. For the central edge-reconstruction scheme, the discretization errors are highly oscillatory spatially and the convergence of discretization error norms is highly erratic. The convergence of the discretization errors is apparently less than first order, but nonetheless the errors converge in all norms. The erratic 
behavior of the discretization errors and insufficient grid refinement may have led to an incorrect conclusion in [18].

Remark on mixed grids computations. Finite-volume computations on general mixed-element grids have been demonstrated in $[9,20]$ for the Euler equations. The results showed $O(1)$ convergence of truncation errors and first order convergence of discretization errors in all norms. On mixed-element grids, with non-smooth interfaces between two regions of regular grids, such as in [18], convergence in integral norms was even better, approaching second order in the $L_{2}$ norm. Zeroth order convergence of the $L_{\infty}$ norms of the discretization errors and partial-order convergence of the $L_{2}$ norms of the discretization errors reported in [18] for advection computations on mixed grids is attributed to insufficient refinement and the erratic convergence of discretization errors characteristic for central schemes with checkerboard instabilities.

5. Conclusions. Truncation-error analysis is a reliable tool in predicting convergence rates of discretization errors on regular smooth grids. However, it is often misleading in application to finite-volume discretization schemes on irregular (e.g., unstructured) grids. Convergence of truncation errors severely degrades on general irregular grids; a design-order convergence can be achieved only on grids with a certain degree of geometric regularity. Such degradation of truncation-error convergence does not necessarily imply a lower-order convergence of discretization errors. In these notes, irregular-grid computations with finite-volume discretization schemes demonstrate that the design-order discretization-error convergence can be achieved even when truncation errors exhibit a lower-order convergence or, in some cases, do not converge at all.

\section{REFERENCES}

[1] M. Aftosmis, D. Gaitonde, and T. S. Tavares, Behavior of linear reconstruction techniques on unstructured meshes, AIAA Journal, 33 (1995), pp. 2038-2049.

[2] W. K. Anderson And D. L. Bonhaus, An implicit upwind algorithm for computing turbulent flows on unstructured grids, Computers and Fluids, 23 (1994), pp. 1-21.

[3] S. BARBeiro, Supraconvergent cell-centered scheme for two dimensional elliptic problems, Appl. Num. Math, 59 (2009), pp. 56-72.

[4] T. J. BARTH, Numerical aspects of computing high-Reynolds number flow on unstructured meshes, AIAA Paper 91-0721, 29-th AIAA Aerospace Science Meeting, Reno, NV, January 1991.

[5] - Recent developments in high-order k-exact reconstruction on unstructured meshes, AIAA Paper 93-0668, 31-st AIAA Aerospace Science Meeting, Reno, NV, January 1993.

[6] D. Bouche, J.-M. Ghidaglia, And F. Pascal, Error estimate and the geometrical corrector for the upwind finite volume method applied to the linear advection equation, SIAM J. Numer. Anal., 43 (2005), pp. 578-603.

[7] B. Despres, Lax theorem and finite volume schemes, Mathematics of Computations, 73 (2003), pp. 1203-1234.

[8] B. Diskin And J. L. Thomas, Accuracy analysis for mixed-element finite-volume discretization schemes, tech. rep., NIA Report 2007-08, National Institute of Aerospace, August 2007.

[9] B. Diskin, J. L. Thomas, E. J. Nielsen, H. Nishikawa, and J. A. White, Comparison of node-centered and cell-centered unstructured finite-volume discretizations. Part I: viscous fluxes, AIAA Paper 2009-597, 47-th AIAA Aerospace Sciencies Meeting, Orlando, FL, January 2009.

[10] M. B. GILES, Accuracy of node-based solutions on irregular meshes, in 11-th International Conference on Numerical Methods in Fluid Dynamics, D. L. Dwoyer, M. Y. Hussaini, and R. Voigt, eds., Lecture Notes in Physics, v. 323, Springer-Verlag, 1989, pp. 369-373.

[11] A. C. Haselbacher, A Grid-Tranparent Numerical Method for Compressible Viscous Flow on Mixed Unstructured Meshes, PhD thesis, Loughborough University, 1999.

[12] A. C. Haselbacher, J. J. McGuirk, and G. J. Page, Finite-volume discretization aspects for viscous flows on mixed unstructured grids, AIAA Journal, 37 (1999), pp. 177-184. 
[13] H.-O. Kreiss, T. A. Manteuffel, B. Wendroff, and J. A. B. White, Supra-convergence schemes on irregular grids, Mathematics of Computation, 47 (1986), pp. 537-554.

[14] D. R. Lindquist And M. B. Giles, A comparison of numerical schemes on triangular and quadrilateral meshes, in 11-th International Conference on Numerical Methods in Fluid Dynamics, D. L. Dwoyer, M. Y. Hussaini, and R. Voigt, eds., Lecture Notes in Physics, v. 323, Springer-Verlag, 1989, pp. 273-277.

[15] T. A. Manteuffel And A. B. White, The numerical solution of the second-order boundary value problem on nonuniform meshes, Mathematics of Computations, 47(176) (1986), pp. 511-536.

[16] F. PASCAL, On supra-convergence of the finite volume method for the linear advection, ESIAM: Proc., 18 (2007), pp. 38-47.

[17] P. L. RoE, Error estimates for cell-vertex solutions of the compressible Euler equations, ICASE Report 87-6, NASA CR-1987-178235, January 1987.

[18] M. SvÄrd, J. Gong, AND J. NordStröm, An accuracy evaluation of unstructured nodecentered finite-volume methods, Appl. Num. Math., 58 (2008), pp. 1142-1158.

[19] A. Syrakos And A. Goulas, Estimate of the truncation error of finite volume discretization of the Navier-Stokes equations on collocated grids, Int. J. Numer. Meth. Fluids, 50 (2006), pp. 103-130.

[20] J. L. Thomas, B. Diskin, And C. L. Rumsey, Towards verification of unstructured-grid solvers, AIAA Journal, 46 (2008), pp. 3070-3079.

[21] A. N. Tikhonov and A. A. Samarsky, Homogeneous difference schemes on nonuniform nets, Zh. Vychsl. Mat. and Mat. Fiz., 2 (1962), pp. 812-832. English translation in U.S.S.R. Comput. Math. and Math. Phys., pp. 927-953.

[22] E. Turkel, Accuracy of schemes with nonuniform meshes for compressible fluid flows, Appl. Numer. Math., 2 (1986), pp. 529-550. 